\title{
Lipid Oligonucleotide Conjugates as Responsive Nanomaterials for Drug Delivery
}

\author{
Oleksandr Pokholenko, ${ }^{a, b, e}$ Arnaud Gissot, ${ }^{a, b, e}$ Brune Vialet,${ }^{a, b}$ Katell Bathany, ${ }^{c}$ Alain Thiery, ${ }^{d}$ and Philippe \\ Barthélémy*a,b
}

\section{Received (in $X X X, X X X)$ Xth $X X X X X X X X X 20 X X$, Accepted Xth XXXXXXXXX 20XX DOI: 10.1039/b000000x}

We report Lipid OligoNucleotide conjugates (LONs) bearing either two or three hydrophobic chains. LONs self-assemble into micellar aggregates, which provide a suitable reservoir ${ }_{10}$ for hydrophobic drug such as paclitaxel. Our results demonstrate that the composition of the LONs both in terms of the lipid and the oligonucleotide sequence impact their ability to host lipophilic molecules. Interestingly, binding of the complementary oligonucleotide selectively induces the 15 release of part of the drug payload of the aggregates. These LON based micelles, which efficiently host hydrophobic drugs represent an original stimuli-responsive drug delivery system.

\section{INTRODUCTION}

The ability of DNA to form predictable and complexe nano20 architectures is virtually unlimited. ${ }^{1}$ Recently, much attention has been given to Lipid-OligoNucleotide bioconjugates (LONs). ${ }^{2,3}$ These amphiphiles feature an oligonucleotide sequence as the polar head and at least one lipid moiety inserted either at the 3'or 5'-end of the oligonucleotide or within the sequence. 25 Interestingly, LONs self assemble to give aggregates such as micelles $^{4-6}$ and vesicles. ${ }^{7,8}$ Shape-shifting "smart" LONs have also been described. ${ }^{9-11}$

The appended lipidic segment of LONs brings about new properties to these surfactants like enhanced antisense activities, ${ }^{12}$ 30 tagging of vesicles ${ }^{13-17}$ or lipid bilayers, ${ }^{4,18-21}$ and biological membranes. ${ }^{22-24}$ LONs have also been successfully implemented in original detection schemes for nucleic acids, ${ }^{25,26}$ and as biotechnological tools. ${ }^{27,28}$ Besides, LON aggregates feature a lipophilic core or reservoir, which can be used for the loading of 35 hydrophobic drugs. We hypothesized that the drug loading of the aggregates could be modulated via the hybridization of the complementary ON sequence, thus making these LON aggregates an attractive original nanomedicine. ${ }^{29}$ Ideally, biologically relevant complementary $\mathrm{ON}$ target sequences, as for instance

\footnotetext{
${ }^{a}$ Université Bordeaux Segalen, 146 rue Léo Saignat 33076 Bordeaux Cedex, France. Fax: 3355757 1015; Tel: 3355757 4853;

E-mail: philippe.barthelemy@inserm.fr;

${ }^{b}$ INSERM U869, Bordeaux, F-33076, France.

${ }^{c}$ Université Bordeaux, CBMN - UMR 5248, Pessac F-33600, France.

${ }^{d}$ Université Marseille, IMBE - CNRS UMR 7263, Marseille France.

${ }^{e}$ These authors contributed equally to the work

$\dagger$ Electronic Supplementary Information (ESI) available: Experimental procedures, NMR, MS, TEM, SEM images and DLS data. See http://dx.doi.org/10.1039/b000000x/
}

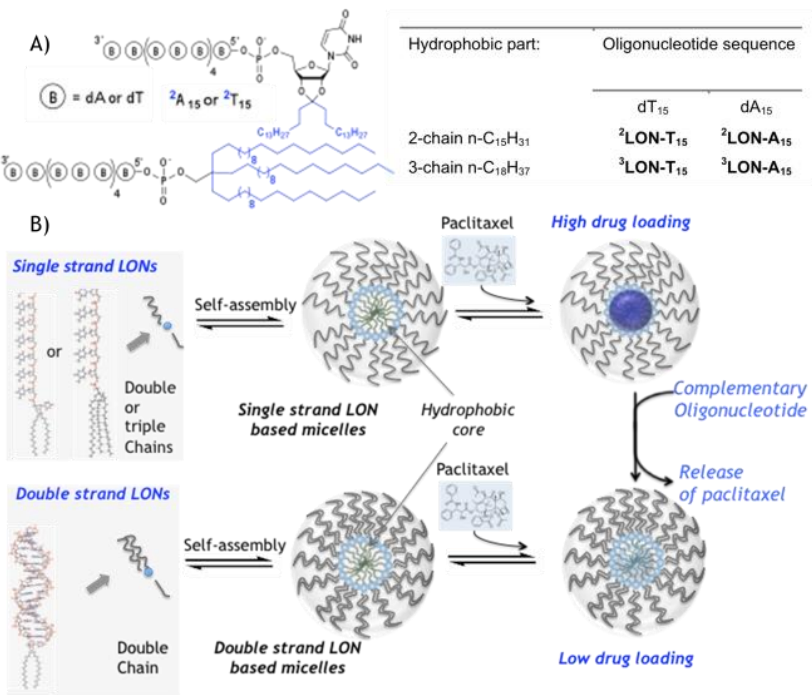

Figure 1. A) Double and triple chain LONs synthetized in this study. B) Schematic representation of single and double strand LON based micelles loaded with an antitumoral drug: Paclitaxel.

40 miRNA, could be used to specifically trigger the release of the drug in vivo.

In the present study, LONs were investigated for their ability to self assemble and load paclitaxel (Figure 1). In order to optimize the inclusion of the lipophilic paclitaxel, LONs were 45 synthesized with either 2 or 3 hydrocarbon chains attached at their respective 5' end (Figure 1A). We show that LON based micelles, which are efficient host for hydrophobic drugs like paclitaxel, can provide stimuli-responsive drug-carriers sensitive to the presence of a complementary oligonucleotide.

50

\section{MATERIALS AND METHODS}

2-1 Synthesis of the triple chain phosphoramidite 4

Details for the experimental procedure for the synthesis of the phosphoramidite $\mathbf{4}$ can be found in the supporting information. Of 55 note, MALDI-MS of the very lipophilic 2,2,2-tris-stearyl acetic acid 3 required the use of a DCTB matrix (trans-2-[3-(4-tertbutylphenyl)-2-methyl-2-propenylidene]malononitrile) doped with $\mathrm{Ag}^{+}$(or $\mathrm{Na}^{+}$).

Procedure: this analysis was performed by the CESAMO ${ }_{60}$ (Bordeaux, France) on a Voyager mass spectrometer (Applied 
Biosystems). The instrument is equipped with a pulsed $\mathrm{N}_{2}$ laser $(337 \mathrm{~nm})$ and a time-delayed extracted ion source. Spectra were recorded in the positive-ion mode using the reflectron and with an accelerating voltage of $20 \mathrm{kV}$. Samples were dissolved in $\mathrm{CH}_{2} \mathrm{Cl}_{2}$ 5 at $10 \mathrm{mg} / \mathrm{ml}$. The DCTB matrix solution was prepared by dissolving $10 \mathrm{mg}$ in $1 \mathrm{ml}$ of $\mathrm{CH}_{2} \mathrm{Cl}_{2}$. A solution of cationisation agent (AgTFA, $10 \mathrm{mg} / \mathrm{ml}$ ) was also prepared. The solutions were combined in a 10:1:1 volume ratio of matrix to sample to cationisation agent. One to two microliters of the obtained 10 solution was deposited onto the sample target and vacuum-dried.

\section{2-2 LON synthesis and MS}

LONs were synthesized using the phosphoramidite methodology on an automated Expedite 8909 DNA synthesizer at the $\mu$ mole scale on $500 \AA$ primer support (loading: $60-100 \mu \mathrm{mol} / \mathrm{g}$, Link 15 technologies, Synbase Control Pore Glass). Autoclaved milliQ water has been used whenever water was required for the handling of oligonucleotides or LONs.

Mass spectra measurements were performed on a MALDI-TofToF mass spectrometer (Ultraflex, Bruker Daltonics, Bremen, ${ }_{20}$ Germany). Best results were obtained in the linear mode with positive-ion detection. Mass spectra were acquired with an ion source voltage 1 of $25 \mathrm{kV}$, an ion source voltage 2 of $23.5 \mathrm{kV}$, a lens voltage of $6 \mathrm{kV}$, by accumulating the ion signals from 1000 laser shots at constant laser fluence with a $100 \mathrm{~Hz}$ laser. External 25 mass calibration was achieved using a mixture of oligonucleotides $\mathrm{dT}_{12}-\mathrm{dT}_{18}$ (Sigma).

1:1 mixture of samples of LONs $(\sim 20-50 \mu \mathrm{M})$ and matrix was spotted on a MALDI target and air-dried before analysis.

The performance of the following matrices was evaluated: 2,530 dihydroxybenzoic acid (DHB), 2,4,6-trihydroxy-acetophenone (THAP), 3-hydroxypicolinic acid (3-HPA) and 2,6dihydroxyacetophenone (DHA). THAP at a concentration of 20 $\mathrm{mg} / \mathrm{mL}$ in a $4: 1$ mixture of ethanol and $100 \mathrm{mM}$ aqueous ammonium citrate was shown to yield optimal mass spectral 35 results.

2-3 Transmission and Scanning Electron Microscopy (TEM and SEM)

Transmission Electron Microscopy (TEM). Samples were imaged by negative staining microscopy with a Hitachi H 7650 40 electron microscope. Samples containing LONs (concentrations and mean sizes are indicated in TEM images) were transferred to a carbon-coated copper grid for ten minutes. The sample was then dried and stained with $2.5 \%(\mathrm{~W} / \mathrm{W})$ of uranyl acetate in water for five minutes.

${ }_{45}$ Scanning Electron Microscopy (SEM). Samples were examined at an accelerating voltage of $10 \mathrm{kV}$. Typically $20 \mathrm{ml}$ of sample containing the LON aggregates were dried spread on polished stainless steel platelets and then coated with a 6-10 nm layer of gold-palladium alloy under vacuum with a sputter Coater Balzers ${ }_{50}$ SCD050. Observations were made with a Philips XL30S FEG instrument.

2-4 Solubilization of paclitaxel in aqueous solutions of LONs

Representative example: paclitaxel solubilization by a mixture of ${ }_{55} \mathrm{dA}_{15}$ and $2 \mathrm{LON} \mathrm{T}_{15}$ oligonucleotides.

Preparation of the samples. For the experiments of LONassisted dissolution of paclitaxel, $40 \mathrm{nmol}$ of $\mathrm{dA}_{15}$ and ${ }^{2} \mathbf{L O N}-\mathbf{T}_{15}$ were dried (speedvac). $0.2 \mathrm{~mL}$ PBS (10 mM phosphate buffer $\mathrm{pH}$
$7+100 \mathrm{mM}$ of $\mathrm{NaCl}$ ) were then added and the mixture vortexed. ${ }_{60}$ The obtained aqueous solution was added to a test tube containing $0.6 \mathrm{mg}$ of thoroughly dried paclitaxel. Vortexing and then sonication at $30^{\circ} \mathrm{C}$ for $30 \mathrm{~min}$ produced turbid colloidal suspensions (pure buffer solutions gave clear solutions above the paclitaxel precipitate). Subsequent centrifugation (2000 rpm; ${ }_{65} 20 \mathrm{~min}$ ) followed by filtration through a $450 \mathrm{~nm}$ filter gave the sample ready for HPLC analysis.

For the release of paclitaxel experiments, the procedure was similar to the one described above except that $\mathrm{dA}_{15}$ was not added in the first place. After the ${ }^{2} \mathbf{L O N} \mathbf{T}_{\mathbf{1 5}}+$ paclitaxel solution has 70 been filtered, the latter was added to an equimolar amount of dry dA15 or control oligonucleotide. The mixture was vortexed and left standing at room temperature for $8 \mathrm{~h}$ before the suspension was filtered again. The long period of time left for the hybridization of the 2 oligonucleotides was necessary to leave 75 time for the released paclitaxel molecules to aggregate in large filterable particles.

Determination of paclitaxel concentrations. The concentrations of paclitaxel in the different aqueous solutions were measured by reverse phase (Macherey Nagel EC 250/4 Nucleosil 120-5 C4) ${ }_{80} \mathrm{HPLC}$ with detection at $227 \mathrm{~nm}$. Elution conditions (eluent A $(\mathrm{V} / \mathrm{V}): 5 \% \mathrm{CH}_{3} \mathrm{CN} / 95 \% 0.1 \mathrm{M}$ TEAA pH 7; eluent B: $80 \%$ $\mathrm{CH}_{3} \mathrm{CN} / 20 \% 0.1 \mathrm{M}$ TEAA $\mathrm{pH}$ 7): isochratic (16\% of B) for 2 mns, $16->90 \%$ of $\mathrm{B}$ after $28 \mathrm{mns}$ and back to $16 \%$ of $\mathrm{B}$. A representative chromatogram is included in the supplementary 85 information.

The paclitaxel concentrations were estimated by measuring the area of the paclitaxel peak in the chromatogram after injection of a fixed volume $(45 \mu \mathrm{L})$ of the solution. The latter was translated into a paclitaxel concentration using a calibration curve (see SI). 90 The concentrations tested for the calibration curve spanned the range of paclitaxel concentrations obtained in our paclitaxelloading experiments.

\section{RESULTS AND DISCUSSION}

95 3-1 Chemistry of LONs. LONS were efficiently synthesized using the classical phosphoramidite chemistry for oligonucleotides (see supporting information). While ${ }^{2} \mathbf{L O N}-\mathbf{A}_{15}$ and ${ }^{2} \mathbf{L O N}-\mathbf{T}_{15}$ were synthesized via the phosphoramidite derived from the palmitone ketal of uridine as described before ${ }^{14}$ the 100 synthesis of the second triple hydrocarbon chain modifier is illustrated in Scheme 1 starting from diethyl malonate and stearyl bromide. Malonic ester synthesis first afforded the bis-stearyl

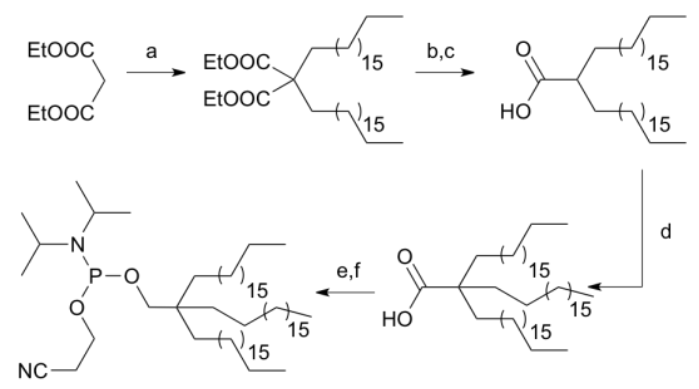

Scheme 1 Synthesis of the triple chain phosphoramidite synthon Reagents and conditions: a) $\mathrm{NaH}$, stearyl bromide, dry DMF, $0-50^{\circ} \mathrm{C}$, $16 \mathrm{~h}$ b) $\mathrm{KOH}, \mathrm{iPrOH} / \mathrm{H}_{2} \mathrm{O}, 80^{\circ} \mathrm{C}, 16 \mathrm{~h}$ c) aq. $\mathrm{H}_{2} \mathrm{SO}_{4}, 77 \%, 2$ steps d) LDA, stearyl bromide, dry THF, RT- $50^{\circ} \mathrm{C}, 68 \%$ e) LAH, dry THF, $60^{\circ} \mathrm{C}$ f) $\mathrm{N}(\mathrm{iPr})_{2} \mathrm{P}(\mathrm{OCNE}) \mathrm{Cl}$, dry $\mathrm{CH}_{2} \mathrm{Cl}_{2}, \mathrm{RT}$ 


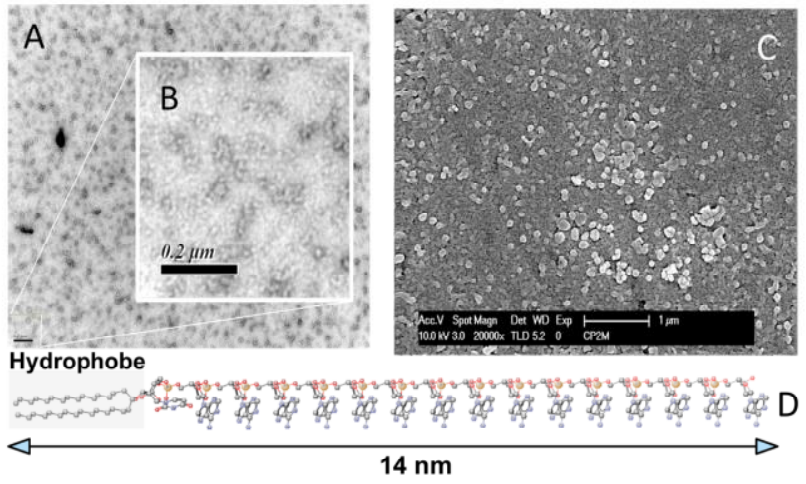

Figure 2. Examples of TEM (A, and magnification B) and SEM (C) images observed with ${ }^{2} \mathbf{L O N}-\mathbf{A}_{15}(\mathrm{C}=40 \mu \mathrm{M}$ in water). Drawings showing i) the dimensions of ${ }^{2} \mathbf{L O N}-\mathbf{A}_{15}$ estimated in the maximum elongated conformation (D). The molecule of ${ }^{\mathbf{2}} \mathbf{L O N}-\mathbf{A}_{15}$ was constructed using MarvinSketch software and optimized in Dreiding force field, see SI for details.

adduct 1, which was further hydrolyzed and decarboxylated to the monoacid 2. The latter was then alkylated with stearyl bromide after deprotonation with an excess of LDA to give the triple chain monoacid 3. Of note and probably because of its high 5 lipophilicity, 3 proved quite reluctant to traditional mass analysis (ESI and maldi using conventional organic matrices). Mass analysis (maldi) of this compound required the use of a DCTB matrix (trans-2-[3-(4-tert-butylphenyl)-2-methyl-2propenylidene]malononitrile) doped with $\mathrm{Ag}^{+}$or $\mathrm{Na}^{+}$(SI).

10 Reduction with LAH followed by phosphitylation of the resulting alcohol afforded the target phosphoramidite $\mathbf{4}$ ready for the automated, solid supported synthesis of oligonucleotides. Oligonucleotides $\mathrm{dT}_{15}$ and $\mathrm{dA}_{15}$ were derivatized with these 2 phosphoramidites (Figure 1A). The coupling efficiency of the 15 new phosphoramidite $\mathbf{4}$ to the elongating oligonucleotide sequence could not be directly determined due to the absence of the DMT protecting group in this phosphoramidite. Nevertheless, the coupling yield was good as estimated by the integration of the mass spectrum signals from the crude reaction mixture of ${ }^{3} \mathbf{L O N}$ -

${ }_{20} \mathbf{T}_{15}$ (see SI) and the excellent $40 \%$ final yield of the HPLCpurified LON. The oligonucleotide sequences $\mathrm{dA}_{15}$ (adenine 15mer) and $\mathrm{dT}_{15}$ (thymidine 15-mer) were appended to these 2 lipophilic modifiers to probe the influence of the oligonucleotide sequence. This resulted in the synthesis of four different LONs 25 (Figure 1A).

3-2 Physicochemical properties of LONs. As true surfactants, LONs can self assemble in aqueous media. Both the lipophilic segment and the oligonucleotide sequence were found 30 to markedly influence the aggregation behavior of our LONs. Overall and not surprisingly, adenosine-containing LONs $\left({ }^{3} \mathbf{L O N}\right.$ $\mathbf{A}_{15}$ and ${ }^{2} \mathbf{L O N}-\mathbf{A}_{15}$ ) were less soluble in water than their

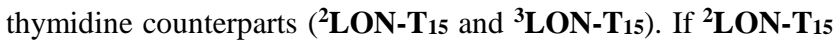
aqueous solutions did not show any sign of precipitation after 35 HPLC purification and storage, ${ }^{\mathbf{2}} \mathbf{L O N}-\mathbf{A}_{15}$ was only sparingly soluble in water (up to ca. $50 \mu \mathrm{M}$ ). Yet, addition of a buffer (PBS or AcOH/TEA) resulted in a better solubilisation of ${ }^{2} \mathbf{L O N}-\mathbf{A}_{15}$. Interestingly, the presence of the triple chain motif resulted in a

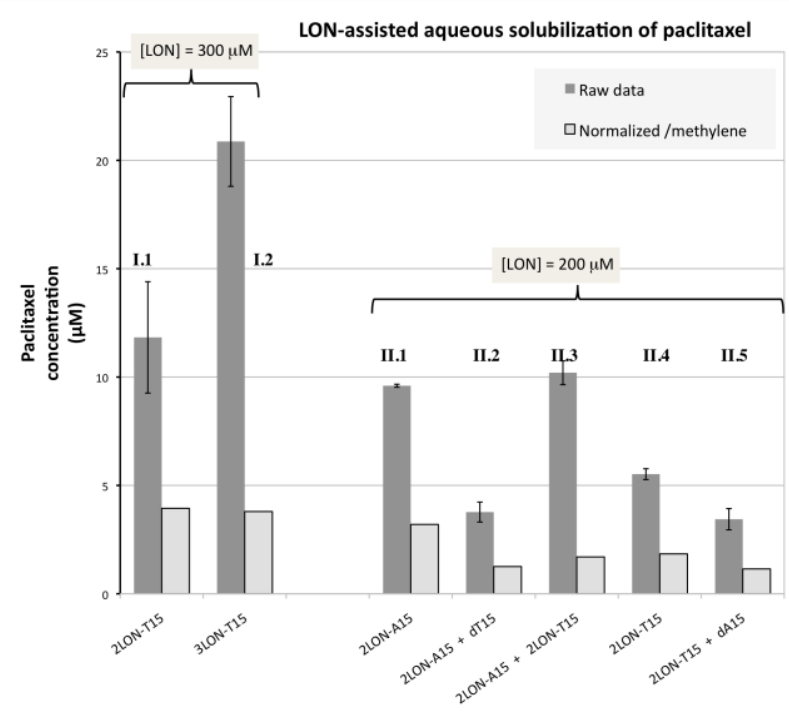

Figure 3. LON-assisted aquous solubilization of paclitaxel in $10 \mathrm{mM}$ phosphate buffer and $100 \mathrm{mM} \mathrm{NaCl}$. Oligonucleotide duplexes (when considered) were annealed prior to the addition of paclitaxel.

peculiar behavior of ${ }^{3} \mathbf{L O N}-\mathbf{A}_{15}$ and ${ }^{3} \mathbf{L O N}-\mathbf{T}_{15}$. The latter could 40 be HPLC-purified and characterized by MS. Yet, a slow aggregation tendency was observed after storage at $-20^{\circ} \mathrm{C},{ }^{3} \mathbf{L O N}$ T15 being still detectable by MS in the solution (see SI). In contrast, immediate and irreversible aggregation of ${ }^{3} \mathbf{L O N}-\mathbf{A}_{15}$ took place after cleavage and deprotection of the crude LON. The 45 aggregates were so stable that MS detection and purification of ${ }^{3} \mathbf{L O N}-\mathbf{A}_{15}$ by PAGE or HPLC proved impossible (see SI). Consequently, dynamic light scattering, transmission electron microscopy and SEM experiments were carried out with the solutions of the 3 purified LONs only. Aqueous samples 50 containing LONs were studied under different conditions including pure water and aqueous suspensions of varying ionic strength and temperature $\left(25-45^{\circ} \mathrm{C}\right)$. All the LONs investigated, namely ${ }^{2} \mathbf{L O N}-\mathbf{T}_{15},{ }^{2} \mathbf{L O N}-\mathbf{A}_{15}$ and ${ }^{3} \mathbf{L O N}-\mathbf{T}_{15}$, yielded small spherical objects ranging from approximately 8 to $20 \mathrm{~nm}$ in 55 diameter as characterized by DLS, transmission and scanning electronic microscopies (TEM and SEM, see SI). For example, TEM and SEM imaging of ${ }^{2} \mathbf{L O N}-\mathbf{A}_{15}$ samples $(\mathrm{C}=40 \mu \mathrm{M})$ dissolved in pure water showed micellar aggregates (Figures $2 \mathrm{~A}$ and 2B). The micellar architecture of these assemblies can be 60 explained based upon the molecular dimensions of 15 nucleobase-long LONs (Figure 2C). Indeed, such molecules are flexible, with cone shape structure and dimensions compatible with the self-aggregation into spherical micelles. The DLS study of ${ }^{2} \mathbf{L O N}-\mathbf{T}_{15}$ in water indicates that the size of aggregates is ${ }_{65}$ poorly sensitive to ionic strength. Likewise, the size of aggregates is poorly affected by temperature in the $25-45^{\circ} \mathrm{C}$ range or the concentration of the amphiphile (see SI).

3-3 Drug loading experiments. Physicochemical experiments 70 and microscopy observations demonstrated that LONs selfassociate into micelle-like aggregates. We hypothesized that the hydrophobic core of LON aggregates could promote the solubilization in aqueous environments of hydrophobic drugs like paclitaxel. To this end, the LONs were solubilized (200 or 300 
$\mu \mathrm{M}$ solutions) in $10 \mathrm{mM}$ PBS buffer at $\mathrm{pH} 7$ and $100 \mathrm{mM} \mathrm{NaCl}$. The LON aqueous solutions were bath-sonicated and heated in the presence of a thin film of paclitaxel and the resulting suspensions filtered through a $0.4 \mu \mathrm{m}$ pore to remove non5 incorporated paclitaxel aggregates. The paclitaxel concentrations still remaining in solution were then determined by RP-C 4 -HPLC (see SI and figure 3). On one hand and as expected, the more lipophilic ${ }^{3} \mathbf{L O N}-\mathbf{T}_{\mathbf{1 5}}$ was superior to ${ }^{\mathbf{2}} \mathbf{L O N}-\mathbf{T}_{\mathbf{1 5}}$ in the solubilization of paclitaxel (Figure 3, compare I.1 and I.2). Yet, 10 when normalized by the number of methyl and methylene groups present in their structure (30 and 55 for ${ }^{2} \mathbf{L O N}-\mathbf{T}_{15}$ and ${ }^{3} \mathbf{L O N}-\mathbf{T}_{15}$ respectively), these two LONs exhibit the same ability to solubilize paclitaxel (light grey bars in I.1 and I.2). For a given oligonucleotide sequence, this seems to indicate that the 15 solubility of paclitaxel is proportional to the number of carbon atoms present in the hydrocarbon chains of LONs. On the other hand, the oligonucleotide sequence was quite unexpectedly found to be of prime importance (compare results II.1 and II.4 in Figure 3): ${ }^{2} \mathbf{L O N}-\mathbf{A}_{15}$ turned out to be almost twice as potent as ${ }^{2} \mathbf{L O N}$ ${ }_{20} \mathbf{T}_{15}$ at solubilizing paclitaxel. The unmodified $\mathrm{dA}_{15}$ and $\mathrm{dT}_{15}$ oligonucleotides being both very poor at solubilizing paclitaxel (results not shown), cooperativity somehow exists between the $\mathrm{dA}_{15}$ and the alkyl segments of ${ }^{2} \mathbf{L O N}-\mathbf{A}_{15}$ to host more drug molecules. Besides, when ${ }^{2} \mathbf{L O N}-\mathbf{A}_{15}$ and ${ }^{2} \mathbf{L O N}-\mathbf{T}_{15}$ ${ }_{25}$ complementary single strand DNA (cDNA: $\mathrm{dT}_{15}$ and $\mathrm{dA}_{15}$ respectively) were allowed to hybridize before the paclitaxel solubilizing step, the paclitaxel intake clearly decreases with both ${ }^{2} \mathbf{L O N}-\mathbf{A}_{15}$.dT 15 and ${ }^{2} \mathbf{L O N}-\mathbf{T}_{15}$.dA 15 aggregates compared to single-strand (ss) LON (compare results II.1/II.2 and II.4/II.5 30 respectively). If this decrease remained modest (37\%) for ${ }^{2} \mathbf{L O N}$ $\mathbf{T}_{15 . d A_{15}}$ aggregates, it reached $61 \%$ for ${ }^{2} \mathbf{L O N}-\mathbf{A}_{15} . \mathrm{dT}_{15}$ micelles. Eventually, the paclitaxel payload of ${ }^{2} \mathbf{L O N}-\mathbf{A}_{15}$ and ${ }^{2} \mathbf{L O N}-\mathbf{T}_{15}$ aggregates reached the same level (within experimental errors) upon binding the complementary oligonucleotides (compare II.2 35 and II.5). In other words, the $\mathrm{dA}_{15}$ segment of ${ }^{2} \mathbf{L O N}-\mathbf{A}_{15}$ is no longer capable of promoting paclitaxel intake once it is engaged into double-strand (ds) DNA. The importance of the $\mathrm{dA}_{15}$ segment is further illustrated by the paclitaxel loading capability of a $1: 1{ }^{2} \mathbf{L O N}-\mathbf{A}_{15}:{ }^{2} \mathbf{L O N}-\mathbf{T}_{15}$ mixture (Figure 3, II.3). If the 40 global payload of the drug increases in that case, this result is merely the result of the doubling of the effective concentration of alkyl chains. When normalized by the number of methylene groups, one clearly sees that this mixture is indeed less likely to host paclitaxel molecules compared to ${ }^{2} \mathbf{L O N}-\mathbf{A 1 5}$ alone (compare 45 grey bars in II.1 and II.3). Interestingly, the drug loading capability of that mixture is within experimental errors equivalent to ${ }^{2} \mathbf{L O N}-\mathbf{T}_{15}$ (compare II.3 and II.4). The benefit of having the $\mathrm{dA}_{15}$ sequence in ${ }^{\mathbf{2}} \mathbf{L O N}-\mathbf{A}_{15}$ is therefore abolished upon annealing of the complementary oligonucleotide.

50

3-4 Controlled release of paclitaxel. If ds-LONs are clearly less efficient at solubilizing paclitaxel in aqueous buffered solutions compared to ${ }^{2} \mathbf{L O N}-\mathbf{A}_{15}$ or ${ }^{2} \mathbf{L O N}-\mathbf{T}_{15}$ micellar aggregates, the controlled release of the paclitaxel drug molecules 55 upon annealing of the cDNA has not been evidenced so far. Despite its lower ability to host paclitaxel molecules, these experiments were conducted with ${ }^{\mathbf{2}} \mathbf{L O N}-\mathbf{T}_{\mathbf{1 5}}$ micellar aggregates for their lesser tendency toward precipitation with time (see the

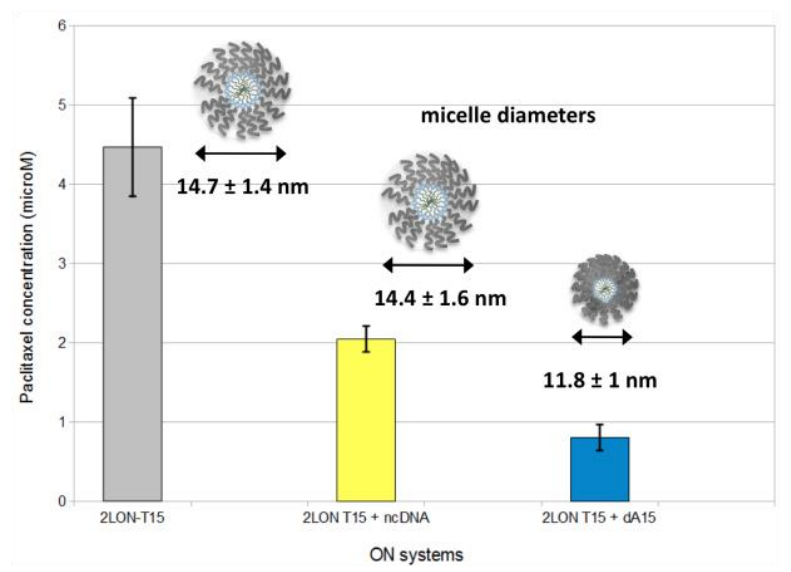

Figure 4. Selective controlled release of paclitaxel from ${ }^{2} \mathbf{L O N}-\mathbf{T}_{15}$ micelles $(200 \mu \mathrm{m}$ based on the LON) upon treatment with the cDNA (10 $\mathrm{mM}$ phosphate buffer $\mathrm{pH} 7,100 \mathrm{mM} \mathrm{NaCl}$ ). The measured (by Dynamic Light Scattering in the absence of paclitaxel) mean size of the micellar aggregates is reported on top for each of the oligonucleotide system reported.

physicochemical properties part). Schematically, the filtered ${ }_{60}$ micellar suspensions of ${ }^{2} \mathbf{L O N}-\mathbf{T}_{15}$ loaded with paclitaxel was subjected either to the cDNA dA 15 or the non complementary ssDNA (5'-GGC TCA CAA CAG GC-3') as a control and the released paclitaxel was again filtered off through $0.4 \mu \mathrm{m}$ pores (Figure 4). While some paclitaxel molecules were non${ }_{65}$ specifically released from the ${ }^{\mathbf{2}} \mathbf{L O N}-\mathbf{T}_{15}$ micelles upon treatment with the ncDNA (ca. 2-fold decrease in paclitaxel loading), the complementary $\mathrm{dA}_{15}$ induced a 5.6-fold decrease thus demonstrating the selective controlled release of the drug.

70 3-5 Discussions. What drives the release of the drug loaded in the hydrophobic core of the aggregates upon DNA annealing is not obvious at first sight. It must first be noticed that the nature of the LON aggregates is not changed upon binding the cDNA (Figure 4, top) and micellar aggregates are still observed in the 75 presence of the cDNA. Yet, contraction of the micelle was observed following hybridization of the complementary $\mathrm{dA}_{15}$ with ${ }^{2} \mathbf{L O N}-\mathbf{T}_{15}$ aggregates (from 14.7 to $11.8 \mathrm{~nm}$ ). Such contraction of the micellar core was not observed with the ncDNA. These results were quite unexpected. Formation of the ${ }_{80}$ rather long and stiff rod-shaped dsDNA was indeed expected to increase the size of the micelle compared to the quite flexible ssDNA segment of LONs. Interestingly, contraction of the micelle was negligible when ${ }^{3} \mathrm{LON}_{-1} \mathrm{~T}_{15}$ and $\mathrm{dA}_{15}$ were allowed to hybridize (results not shown). This result suggests that the 85 micellar hydrophobic core of the micelle-associated or not with a change in the aggregation number-experience much of the contraction upon DNA duplex formation and may shed light on the mechanism of the triggered release of paclitaxel from LON micelles. In fact, the ss-DNA polar heads of LONs may 90 experience some conformational freedom in the aqueous phase within the LON aggregates. The surface area occupied by each polar head of ss-LONs may be quite large. Consequently, the LON alkyl chains may be poorly packed within the hydrophobic core thus leaving room for hosting hydrophobic guests. In the 
contrary, the stiffness and the rather small cross section of DNA double helices might allow for a better compaction of the alkyl chains within the core of the micelle with the concomitant release of part of the loaded paclitaxel molecules. First attempts to 5 confirm this hypothesis by measuring the surface tension of ${ }^{2}{ }^{2}$ ON-T 15 solutions alone and in the presence of the complementary $\mathrm{dA}_{15}$ were quite unsatisfactory because of slow kinetics and complex adsorption isotherms of the LON at the air/water interface. ${ }^{30,31}$ Further investigations are underway to 10 work around this issue.

\section{CONCLUSIONS}

In this study we present the synthesis of LONs featuring double and triple lipophilic chains. These LONs self-assemble 15 into micelles, which are prone to host paclitaxel molecules within their hydrophobic cores. Our results demonstrate that the composition of the LONs both in terms of the lipid and the oligonucleotide sequence impact their ability to host lipophilic molecules. Interestingly, the drug intake of ${ }^{2} \mathrm{LON}_{-} \mathrm{A}_{15}$ was 20 markedly high compared to other LONs. Overall, the polynucleotide sequence serves three roles: (1) it promotes the transfer of the alkyl chains of the LONs into water, (2) it potentializes the drug intake of the LON aggregates and (3) it can hybridize with the cDNA sequence to trigger the release of the 25 drug. Only two oligonucleotide sequences $\left(\mathrm{dA}_{15}\right.$ and $\left.\mathrm{dT}_{15}\right)$ have been investigated in this study. Consequently, there is still room for further optimization of the ON length and composition.

Numerous stimuli-bioresponsive drug delivery systems have been developed to induce the release of the drug in response to 30 certain stimuli like $\mathrm{pH}$, temperature, redox potential, enzymes, light, and ultrasound. ${ }^{32-34}$ In this contribution we demonstrate that the drug loading of LON based nanomaterials can be modulated via a complementary (hand-made) oligonucleotide stimulus. LON aggregates afford an original and specific stimuli-bioresponsive 35 alternative to address the drug controlled delivery issue. However, extracellular small nucleic acids such as miRNA could be an appropriate biological target of our system. Indeed, although miRNAs exert their functions inside the cell, these and other classes of RNAcan be found in extracellular body fluids. ${ }^{35}$

${ }_{40}$ We hope this oligonucleotide-based strategy for the formulation of lipophilic drugs will and other applications in the realm of drug delivery.

\section{ACKNOWLEDGEMENTS}

45

This work has been supported by the French National Agency (ANR) in the frame of its programme blanc (NANAN project no. ANR-08-NANO-028). The authors thank the Bordeaux Imaging Centre (BIC) for technical assistance during TEM observations. ${ }_{50}$ O.P. and P.B. acknowledge financial support from the Army Research Office.

\section{Notes and references}

\footnotetext{
1 E. Stulz, Chem. Eur. J., 2012, 18, 4456-4469.

552 A. Gissot, M. Camplo, M. W. Grinstaff, and P. Barthélémy, Org. Biomol. Chem., 2008, 6, 1324-1333.

3 A. Patwa, A. Gissot, I. Bestel, and P. Barthélémy, Chem. Soc. Rev., $2011,40,5844-5854$.
}

4 C. Gosse, A. Boutorine, I. Aujard, M. Chami, A. Kononov, E. 60 Cogné-Laage, J.-F. Allemand, J. Li, and L. Jullien, J. Phys. Chem. $B, 2004,108,6485-6497$.

5 H. Liu, Z. Zhu, H. Kang, Y. Wu, K. Sefan, and W. Tan, Chem. Eur. J., 2010, 16, 3791-3797.

6 M. Anaya, M. Kwak, A. J. Musser, K. Müllen, and A. Herrmann, 65 Chem. Eur. J., 2010, 16, 12852-12859.

7 P. M. Dentinger, B. A. Simmons, E. Cruz, and M. Sprague, Langmuir, 2006, 22, 2935-2937.

8 F. Teixeira Jr., P. Rigler, and C. Vebert-Nardin, Chem. Commun., 2007, 1130 .

709 M. P. Thompson, M.-P. Chien, T.-H. Ku, A. M. Rush, and N. C. Gianneschi, Nano Lett., 2010, 10, 2690-2693.

10 M.-P. Chien, A. M. Rush, M. P. Thompson, and N. C. Gianneschi, Angew. Chem. Int. Ed., 2010, 49, 5076-5080.

11 L. Wang, Y. Feng, Z. Yang, Y.-M. He, Q.-H. Fan, and D. Liu, 75 Chem. Commun., 2012, 48, 3715-3717.

12 G. Godeau, C. Staedel, and P. Barthélémy, J. Med. Chem., 2008, 51, 4374-4376.

13 P. A. Beales and T. K. Vanderlick, J. Phys. Chem. A, 2007, 111, 12372-12380.

8014 A. Gissot, C. Di Primo, I. Bestel, G. Giannone, H. Chapuis, and P. Barthélémy, Chem. Commun., 2008, 5550-5552.

15 T. Maruyama, H. Yamamura, M. Hiraki, Y. Kemori, H. Takata, and M. Goto, Colloids Surf., B, 2008, 66, 119-124.

16 P. A. Beales and T. K. Vanderlick, Biophys. J., 2009, 96, 1554 851565 .

17 B. Van Lengerich, R. J. Rawle, and S. G. Boxer, Langmuir, 2010, 26, 8666-8672.

18 I. Pfeiffer and F. Höök, J. Am. Chem. Soc., 2004, 126, 10224 10225 .

9019 I. Pfeiffer and F. Höök, Anal. Chem., 2006, 78, 7493-7498.

20 A. Bunge, A. Kurz, A.-K. Windeck, T. Korte, W. Flasche, J. Liebscher, A. Herrmann, and D. Huster, Langmuir, 2007, 23, $4455-4464$

21 P. A. Beales and T. K. Vanderlick, J. Phys. Chem. B, 2009, 113, $95 \quad 13678-13686$

22 G. G. Borisenko, M. A. Zaitseva, A. N. Chuvilin, and G. E. Pozmogova, Nucleic Acids Res., 2009, 37, e28.

23 M. Loew, R. Springer, S. Scolari, F. Altenbrunn, O. Seitz, J. Liebscher, D. Huster, A. Herrmann, and A. Arbuzova, J. Am. Chem. Soc., 2010, 132, 16066-16072.

24 M. J. Palte and R. T. Raines, J. Am. Chem. Soc., 2012, 134, 62186223.

25 A. Gunnarsson, P. Jönsson, V. P. Zhdanov, and F. Höök, Nucleic Acids Res., 2009, 37, e99.

10526 A. Gunnarsson, P. Sjövall, and F. Höök, Nano Lett., 2010, 10, 732 737.

27 T. Maruyama, T. Hosogi, and M. Goto, Chem. Commun., 2007, 4450.

28 M. Kwak, I. J. Minten, D.-M. Anaya, A. J. Musser, M. Brasch, R. 110 J. M. Nolte, K. Müllen, J. J. L. M. Cornelissen, and A. Herrmann, J. Am. Chem. Soc., 2010, 132, 7834-7835.

29 P. Couvreur, Adv. Drug Delivery Rev., 2013, 65, 21-23.

30 M. Banchelli, F. Betti, D. Berti, G. Caminati, F. B. Bombelli, T. Brown, L. M. Wilhelmsson, B. Nordén, and P. Baglioni, J. Phys. Chem. B, 2008, 112, 10942-10952.

31 M. Banchelli, F. Gambinossi, A. Durand, G. Caminati, T. Brown, D. Berti, and P. Baglioni, J. Phys. Chem. B, 2010, 114, 7348-7358.

32 S. Ganta, H. Devalapally, A. Shahiwala, and M. Amiji, J. Control. Release, 2008, 126, 187-204.

12033 E. Fleige, M. A. Quadir, and R. Haag, Adv. Drug Delivery Rev., 2012, 64, 866-884.

34 X. Huang, X. Jiang, Q. Yang, Y. Chu, G. Zhang, B. Yang, and R. Zhuo, J. Mater. Chem. B, 2013, 1, 1860-1868.

35 A. M. Hoy and A. H. Buck, Biochem. Soc. Trans., 2012, 40, 886125 890. 